Provided for non-commercial research and education use. Not for reproduction, distribution or commercial use.

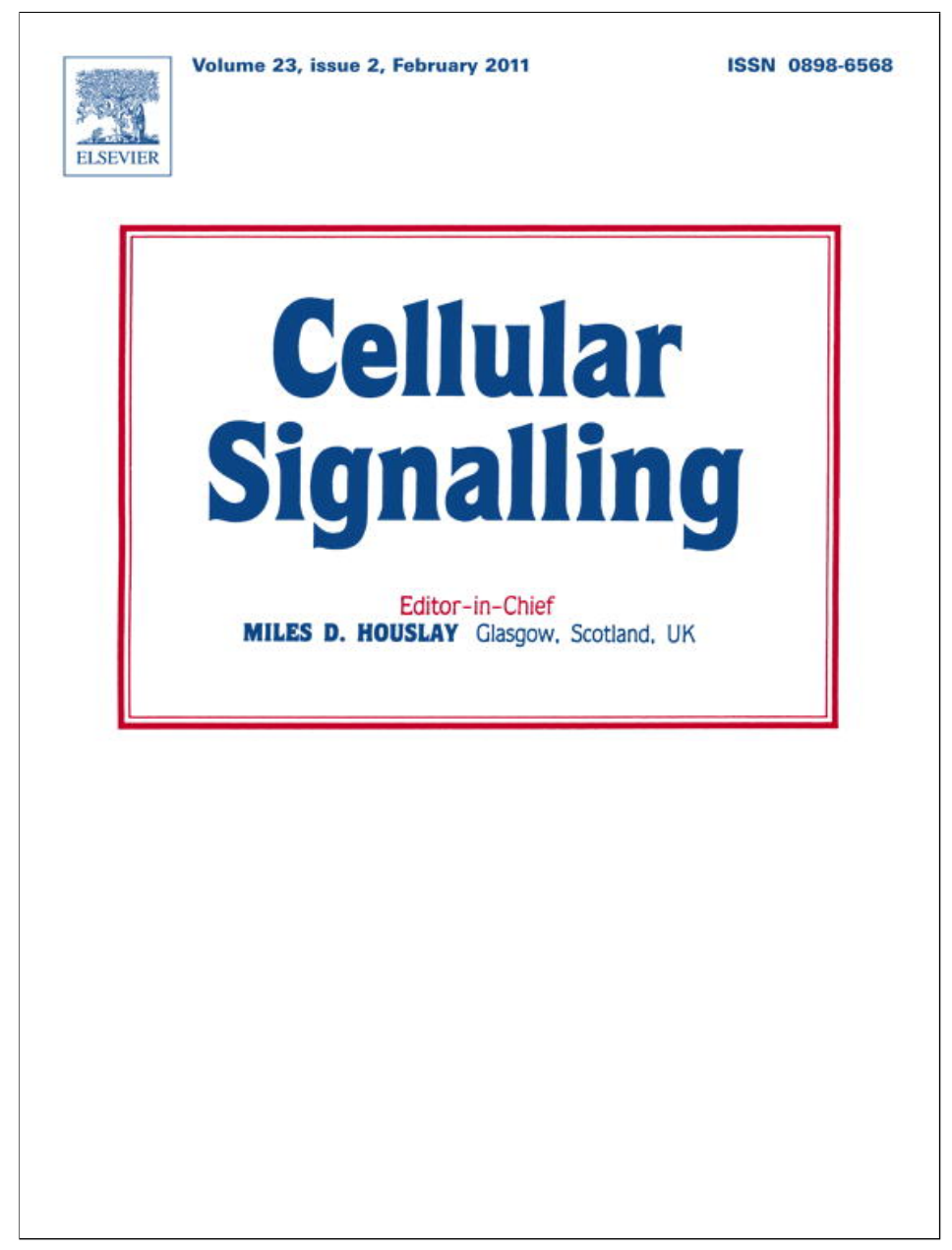

This article appeared in a journal published by Elsevier. The attached copy is furnished to the author for internal non-commercial research and education use, including for instruction at the authors institution and sharing with colleagues.

Other uses, including reproduction and distribution, or selling or licensing copies, or posting to personal, institutional or third party websites are prohibited.

In most cases authors are permitted to post their version of the article (e.g. in Word or Tex form) to their personal website or institutional repository. Authors requiring further information regarding Elsevier's archiving and manuscript policies are encouraged to visit:

http://www.elsevier.com/copyright 


\title{
Bone morphogenetic protein signalling in airway epithelial cells during regeneration
}

\author{
Joanne C. Masterson, Emer L. Molloy, Jennifer L. Gilbert, Natasha McCormack, Aine Adams, Shirley O'Dea* \\ Institute of Immunology, National University of Ireland Maynooth, Maynooth, Ireland
}

\section{A R T I C L E I N F O}

\section{Article history:}

Received 20 August 2010

Accepted 1 October 2010

Available online 16 October 2010

\section{Keywords:}

Airway

Regeneration

Migration

BMP

Smad

1-Nitronaphthalene

\begin{abstract}
A B S T R A C T
Mechanisms of lung regeneration after injury remain poorly understood. Bone morphogenetic protein 4 (BMP4) is critical for lung morphogenesis and regulates differentiation of the airway epithelium during development, although its mechanism of action is unknown. The role of BMPs in adult lungs is unclear. We hypothesised that BMP signalling is involved in regeneration of damaged adult airways after injury. Our aims were to characterise the regeneration process in 1-nitronaphthalene (1-NN) injured airways, to determine if and when BMP signalling is activated during this process and investigate the effects of BMP4 on normal adult airway epithelial cells (AECs). Rats were injected with $50 \mathrm{mg} / \mathrm{kg}$ 1-NN and protein expression in AECs was examined by Western blotting of lung lysis lavage, and by immunofluorescence, at 6, 24, 48 and $96 \mathrm{~h}$ post injection. Expression of signalling molecules p-ERK-1, p-ERK-2 and p-Smad1/5/8 in AECs peaked at $6 \mathrm{~h}$ post injection, coincident with maximal inflammation and prior to airway denudation which occurred at $24 \mathrm{~h}$. While airways were re-epithelialised by $48 \mathrm{~h}$, AEC proliferation peaked later at $96 \mathrm{~h}$ post 1-NN injection. In vitro, BMP4 induced a mesenchymal-like morphology in normal AECs, downregulated E-cadherin expression and increased migration in a wound closure assay. Thus, following acute injury, increased BMP signalling in AECs coincides with inflammation and precedes airway denudation and re-epithelialisation. Our data indicate that, similar to its role in controlling tissue architecture during development, BMP signalling regulates regeneration of the airways following acute injury, involving downregulation of E-cadherin and induction of migration in AECs.
\end{abstract}

(c) 2010 Elsevier Inc. All rights reserved.

\section{Introduction}

Mechanisms underlying regeneration of the airways after injury remain poorly understood. While significant progress has been made in recent years in identifying the progenitor cell types responsible for regenerating the airway epithelium after injury, the signals that control the regeneration process remain unclear. It has been postulated that developmental signals necessary for lung morphogenesis during development may also be involved in regeneration in injured adult lungs but few studies have directly addressed this question [1].

Bone morphogenetic proteins (BMPs) are highly conserved members of the transforming growth factor $\beta$ (TGF $\beta$ ) superfamily and are key regulators of tissue structure both during development and in adult

Abbreviations: 1-NN, 1-Nitronaphthalene; AEC, Airway epithelial cell; BAL Bronchoalveolar lavage; BMP, Bone morphogenetic protein; OVA, Ovalbumin; PCNA, Proliferating cell nuclear antigen; R-Smads, Receptor-regulated Smads; SFM, Serumfree medium; TGF $\beta$, Transforming growth factor $\beta$.

* Corresponding author. Institute of Immunology, National University of Ireland Maynooth, Maynooth, Co. Kildare, Ireland. Tel.: +353 1 7086337; fax: + 3531 7086117.

E-mail addresses: joannemasterson44@gmail.com (J.C. Masterson), emer_molloy@hotmail.com (E.L. Molloy), jengilbert13@gmail.com (J.L. Gilbert), natasha.mccormack@gmail.com (N. McCormack), Aine.Adams@nuim.ie (A. Adams), shirley.odea@nuim.ie (S. O'Dea). organisms. Over 30 different BMP ligands have been identified and shown to influence processes including stem cell maintenance, cell proliferation, migration, differentiation and apoptosis [2]. BMP4 plays a critical role in a variety of developmental processes including epithelialmesenchymal transition (EMT) aspects of morphogenesis [2] and early mesoderm formation [3]. BMP signalling is mediated via type I and type II receptors. Following ligand binding, the type II receptor (BMPR-II) transphosphorylates the type I receptors (BMPR-IA or BMPR-IB) (2). In the canonical pathway, activated type I receptors recruit and phosphorylate the receptor-regulated Smads (R-Smads) 1, 5 and/or 8 . Activated R-Smads form heteromeric complexes with Smad4 and translocate to the nucleus where they act as transcriptional comodulators of specific target genes. Non-canonical signalling also occurs through mitogen-activated protein kinases, phosphoinositide 3-kinase and LIM kinase [4].

BMP4 is essential for lung morphogenesis and also modulates proximal-distal differentiation of the developing epithelium although the mechanism involved is unknown [5]. Few studies have addressed the role of BMP signalling in adult lungs. In a mouse study of ovalbumin (OVA)-induced allergic airway inflammation, BMP signalling was examined at a single time point, $24 \mathrm{~h}$, post-OVA challenge [6]. Immunostaining revealed that all of the BMP type I receptors, including BMPR-IA, were upregulated in inflamed bronchial epithelial cells and induction of BMP2 and BMP4 protein expression was detected in whole lung homogenates from OVA-treated animals. While Smad1 
immunostaining was observed in all bronchial epithelial cells in both control and OVA-treated animals, phosphorylated Smad1/5 (p-Smad1/ 5) was only detected in OVA-treated airways. A study in human asthmatic airways described a somewhat different profile of BMP pathway immunostaining [7]. In contrast to the mouse OVA study, expression of $\mathrm{p}-\mathrm{Smad} 1 / 5$ was detected in both normal and inflamed airway epithelial cells with the incidence reduced in baseline asthmatics and increased following allergen challenge. Therefore, it is unclear whether the canonical BMP signalling pathway is active in normal airway cells and its precise role in health or disease is unknown. We have shown previously that BMP4 downregulates E-cadherin in a transformed airway epithelial cell line [8]. However, it has not yet been established whether normal airway epithelial cells are responsive to BMP2 or BMP4 and the subsequent cellular effects of these ligands are also unknown.

We hypothesise that the key role played by BMP4 in forming the lungs during development is recapitulated in adult lungs during regeneration after injury. Recent reports that BMP signalling regulates epithelial regeneration following damage in the adult zebrafish liver and mouse bladder support this hypothesis $[9,10]$. In the present study, we investigated whether BMP signalling is activated in airway epithelial cells in vivo following acute lung injury and the timing of such activation during regeneration relative to inflammation, epithelial damage and reepithelialisation. For this, we first characterised the chronology of injury/inflammation/regeneration in the 1-nitronaphthalene (1-NN) rat model of acute lung injury. We then used a combination of lung lysis lavage [11] and immunofluorescence to examine BMP signalling specifically within airway epithelial cells. We also determined whether normal airway epithelial cells are responsive to BMP4 and investigated the effects of BMP4 on these cells.

\section{Materials and methods}

\subsection{Animals and 1-nitronapthalene treatment}

All work was carried out in accordance with the EC Directive 86/609/ EEC for animal experiments. All animal use was approved in advance by the Animal Use and Care Committee at the University of California, Davis, USA (UC Davis). Male Sprague-Dawley rats were purchased from Harlan (Indianapolis, IN, USA). Animals were fed and watered ad libitum and housed in an American Association for Accreditation of Laboratory Animal Care accredited facility in HEPA-filtered cage racks at UC Davis for 7 days prior to use. Thirty two rats (225-250 g, aged 8-10 wks) were randomly assigned to eight experimental groups $(n=4)$. Rats were treated by single intraperitoneal injection with $50 \mathrm{mg} / \mathrm{kg}$ body weight 1-nitronapthalene (1-NN) diluted in oleic oil or with oleic oil alone (vehicle control). All treatments were administered between 8 am and $10 \mathrm{am}$. Groups $(\mathrm{n}=4)$ of 1 -NN or vehicle-treated rats were sacrificed at $6,24,48$ and $96 \mathrm{~h}$ following administration of treatments with an overdose intraperitoneal injection of sodium pentobarbital.

2.2. Lung epithelial cell-enriched lysis lavage, bronchoalveolar lavage fluid analysis, lung tissue fixation and haematoxylin and eosin staining

Rat lungs $(\mathrm{n}=3)$ were processed for lysis lavage as described previously [11]. Briefly, rats were sacrificed and the trachea was exposed and cannulated. The lungs were removed from the thoracic cavity and inflated with agarose solution immediately followed by dextrose solution. Both solutions were preheated to $37^{\circ} \mathrm{C}$. The inflated lungs were incubated in a chilled 5\% dextrose bath to allow the agarose to solidify. The dextrose solution was then removed through the cannula and stored on ice. The airways were then lavaged with lysis buffer containing $2 \mathrm{M}$ thiourea, $7 \mathrm{M}$ urea, $4 \% \mathrm{wt} / \mathrm{vol}$ CHAPS, $0.5 \%$ wt/vol Triton X-100, $1 \%$ wt $/$ vol DTT, and $2 \%$ vol $/$ vol protease inhibitor cocktail III (Calbiochem, La Jolla, CA). The epithelial cellenriched lysate was recovered immediately, flash frozen on dry ice and stored at $-80^{\circ} \mathrm{C}$ until use. Protein concentration was determined using the Bradford assay. Total cell counts were obtained from the dextrose solution recovered from the airways during lysis lavage. Rat lungs $(\mathrm{n}=1)$ were fixed in $1 \%$ paraformaldehyde overnight at $4{ }^{\circ} \mathrm{C}$, paraffin embedded and $4 \mu \mathrm{m}$ sections were stained by routine haematoxylin and eosin staining.

\subsection{Primary airway epithelial cell isolation and BMP4 treatment}

Normal primary mouse AECs were harvested from euthanized (sodium pentabarbitol overdose) female $\mathrm{C} 3 \mathrm{H}-\mathrm{Hen}$ mice as described previously [12]. Freshly isolated AECs were seeded onto fibronectincoated glass multiwell chamber slides (Nunc, Roskilde, Denmark) in plating medium (Dulbeco's Modified Essential Medium (Gibco, Glasgow, UK), 10\% fetal bovine serum, $2 \mathrm{mM}$ L-glutamine (Gibco), $100 \mathrm{U} / \mathrm{ml}$ penicillin and $100 \mu \mathrm{g} / \mathrm{ml}$ streptomycin (Gibco)). The following day, cells were rinsed once with serum-free Hams F12 basal medium and then cultured in defined serum-free medium (SFM) (1:1 Hams F12:M199 (Gibco)), $2 \mathrm{mM}$ L-glutamine, $100 \mathrm{U} / \mathrm{ml}$ penicillin, $100 \mu \mathrm{g} / \mathrm{ml}$ streptomycin, $100 \mathrm{U} / \mathrm{ml}$ insulin-transferin-selenium (Gibco), $100 \mathrm{ng} / \mathrm{ml}$ hydrocortisone (Sigma-Aldrich, Dublin, Ireland) and $10 \mathrm{ng} / \mathrm{ml}$ epidermal growth factor (R\&D Systems, Minneapolis, USA). After $24 \mathrm{~h}$, supernatant was removed and fresh SFM was added containing appropriate concentrations of BMP4.

\subsection{Wound closure assay}

The wound closure assay is a modification of the Platypus Technologies Oris Cell Migration Assay (Abingdon, UK). Each well of an Oris 96-well plate was prepared as follows: the centre of the well was coated with $50 \mathrm{ng} / \mathrm{ml}$ fibronectin, allowed to dry for $2 \mathrm{~h}$ and a stopper was then placed in the well to cover the fibronectin; the outer ring of the well was then coated with $50 \mathrm{ng} / \mathrm{ml}$ collagen overnight at $4{ }^{\circ} \mathrm{C}$; excess collagen was removed and primary mouse AECs were seeded in plating medium into this outer area. After 2 days, plating medium was replaced with SFM. On day 5, plugs were removed and medium was replaced with SFM containing $100 \mathrm{ng} / \mathrm{ml} \mathrm{BMP4}$ or $10 \%$ FCS. After $24 \mathrm{~h}$, cells were stained with Giemsa stain (Sigma-Aldrich, Dublin, Ireland). The Oris 'detection mask' was used to visualize the wound area which was photographed and migrated cells were counted using Adobe Photoshop CS3 Extended Cell Count software.

\subsection{Western blotting for rat lung lysis lavage proteins and mouse airway epithelial cell proteins}

Western blotting and densitometric analyses were carried out as previously described [8]. Primary antibodies used were: actin (clone 20-33, Sigma-Aldrich) and pERK, T-ERK and p-Smad1/5/8 (PhosphoSmad1 (Ser463/465)/Smad5 (Ser463/465)/Smad8 (Ser426/428)) (Cell Signalling Technology, Danvers, MA, USA).

\subsection{Immunofluorescence of lung tissue and cultured lung cells}

Lung tissues were deparaffinised in xylene, rehydrated and antigen retrieved by boiling in citrate buffer. Cultured cells were fixed with pre-chilled methanol. Tissue sections and cells were incubated with primary antibodies overnight at $4{ }^{\circ} \mathrm{C}$ followed by appropriate Alexa Fluor ${ }^{\circledR}$ 488-labeled secondary antibodies (Molecular Probes, Invitrogen, Paisley, UK) for $30 \mathrm{~min}$ at room temperature. Nuclei were counterstained with DAPI (Sigma-Aldrich). In addition to antibodies described earlier, primary antibodies used were: PCNA (clone PC 10, Sigma-Aldrich), E-cadherin (clone 36, BD Biosciences, Oxford, UK) and Smad5 (clone D-20) and Smad8 (clone A-17) from Santa Cruz Biotechnology, Santa Cruz, CA, USA. 


\subsection{Quantitative PCR and RT-PCR}

Quantitative PCR for E-cadherin and RT-PCR for Snai1 was carried out as described previously [8]. Samples $(\mathrm{n}=2)$ were run in triplicate and GAPDH was used as the endogenous control.

\section{Results}

3.1. Characterisation of regeneration process in rat airways following 1-nitronaphthalene-induced acute lung injury

The cytotoxicant naphthalene and its derivatives have been widely used to study acute injury mechanisms and to identify stem/progenitor populations in rodent airways [13-15]. Few studies however have used these models to identify the growth factor signals that drive airway regeneration following injury. 1-Nitronaphthalene (1-NN) is considered the most toxic of the naphthalene derivatives and occurs in cigarette smoke, diesel exhaust and in ambient air [16]. Toxicological processes have been described previously in rat 1-NN models with most studies focusing on the 24 hour period post 1-NN exposure and few studies extending to $48 \mathrm{~h}$ [17]. High levels of cytochrome P450 2B1 in rat Clara cells in the airways are thought to bioactivate 1-NN leading to Clara cell death and also death of neighbouring ciliated cells [16]. Cell death is accompanied by mild leukocytic infiltration, however injury remains localised to the epithelium [17]. In order to identify the signals that control regeneration of the airway epithelium after injury, we examined lung regeneration processes in rats exposed to $50 \mathrm{mg} / \mathrm{kg} 1-\mathrm{NN}$ at times up to $96 \mathrm{~h}$ post exposure.

We were interested in determining a time course of BMP signalling relative to inflammation and epithelial injury, proliferation and restoration. Ultrastructural alterations in response to 1-NN up to $96 \mathrm{~h}$ post exposure were examined in haematoxylin and eosin stained tissue sections in order to characterise for the first time the chronology of airway inflammation/injury/regeneration in this model. No histological evidence of epithelial injury was apparent at $6 \mathrm{~h}$ post 1-NN injection (Fig. 1A). However, at $24 \mathrm{~h}$ following administration, substantial airway epithelial damage was observed at all airway levels. The epithelium was discontinuous with regions exhibiting loss of epithelial cells and denudation of the basement membrane. By $48 \mathrm{~h}$ post exposure, the airway surface was re-epithelialised.

The time course of inflammation induced by $1-\mathrm{NN}$-mediated toxicity was examined. Cellular infiltration of the airway lumen was quantified from bronchoalveolar lavage (BAL) fluid at $6,24,48$ and $96 \mathrm{~h}$ post 1 -NN exposure. This infiltrate was maximal at $6 \mathrm{~h}$ following administration of 1 -NN and subsequently decreased over the time course analysed. We now show here that infiltration remained depressed at $96 \mathrm{~h}$ (Fig. 1B).
A

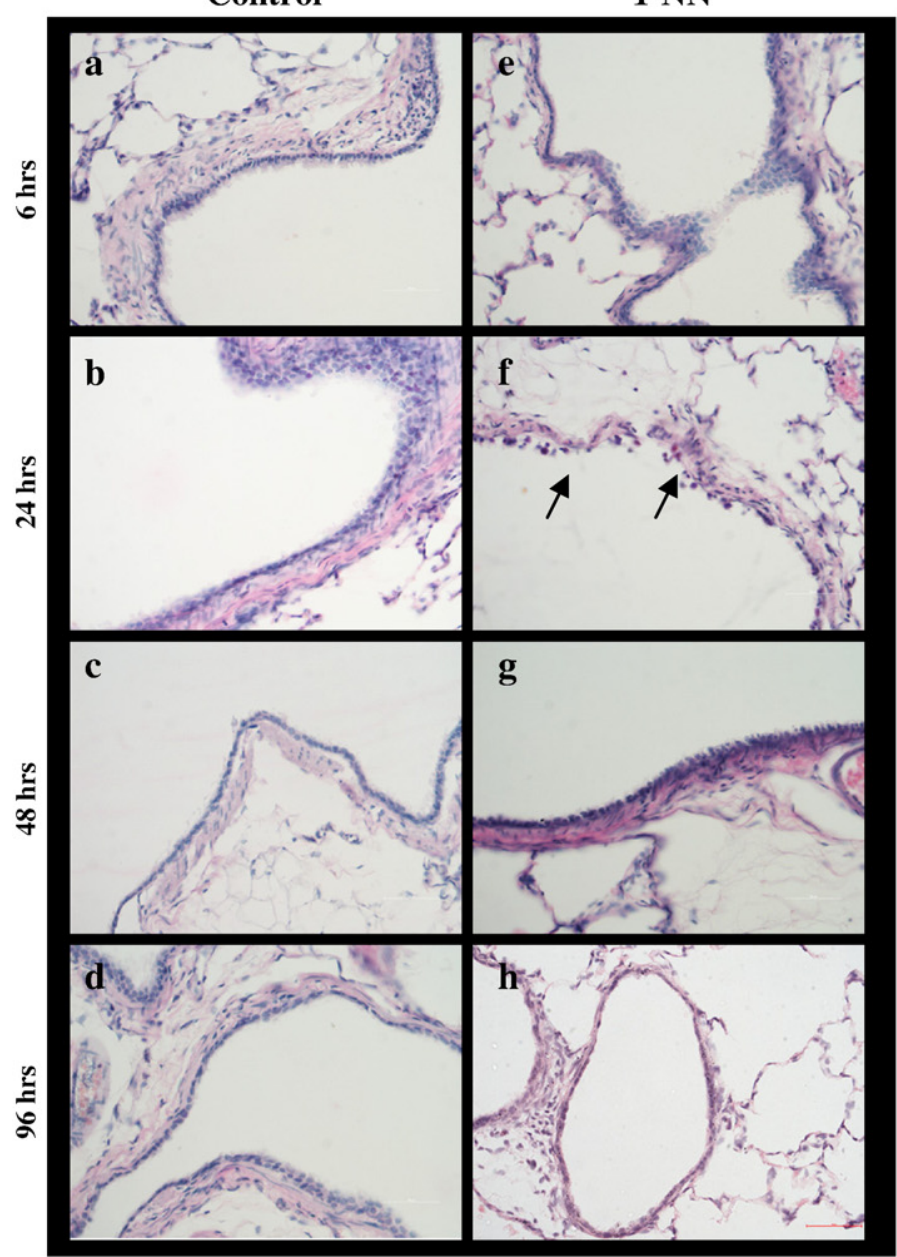

B

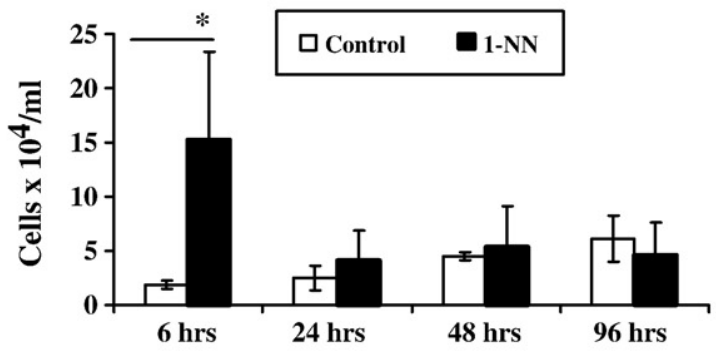

C

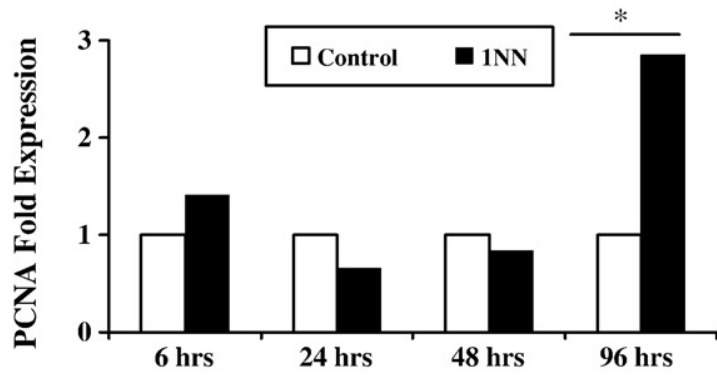

D

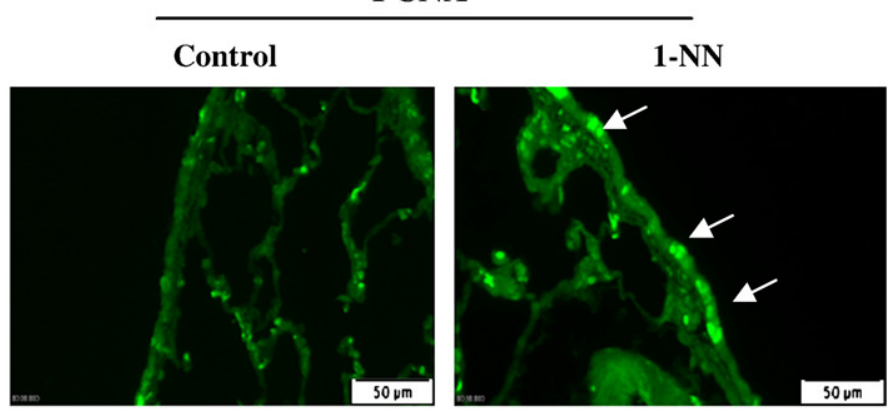

Fig. 1. Time course analysis of injury, inflammation and regeneration in airways of 1-NN-treated animals over $96 \mathrm{~h}$. (A) Histological analysis of haematoxylin and eosin stained control (a-d) and 1-NN-treated (e-h) tissues. At $6 \mathrm{~h}$ post injection, the airway epithelium appeared normal in 1-NN-treated animals (e) while at $24 \mathrm{~h}$, the epithelium was discontinuous with regions exhibiting loss of epithelial cells and denudation of the basement membrane (arrows) (f). At $48 \mathrm{~h}$ and $96 \mathrm{~h}$ post injection, the airways were re-epithelialised. (B) Bronchoalveolar lavage (BAL) fluid infiltrate was maximal at $6 \mathrm{~h}$ post $1-\mathrm{NN}$ injection. Each time point represents $n=3$ except for $48 \mathrm{~h}$ which represents $n=2$. (C) Densitometric analysis of Western blots of lung lysate lavage revealed that PCNA levels were significantly increased 1.8 -fold in AECs in 1-NN-treated animals at $96 \mathrm{~h}$ compared to controls, $\mathrm{n}=3$. (D) Immunofluorescence of tissue sections demonstrated regions of focal PCNA-positive AECs at $96 \mathrm{~h}$. Significance compared to control cells was obtained using the Student's $t$ test ( ${ }^{*} \mathrm{P}<0.05$ ). Scale bars represent $50 \mu \mathrm{m}$. 
To characterise the regeneration process following 1-NN-mediated injury, the timing of airway epithelial cell proliferation was determined by examining proliferating cell nuclear antigen (PCNA) expression. In order to quantify the levels of PCNA specifically within the regenerating airway epithelium, lung lysis lavage was carried out. This technique generates lysates that are highly enriched for AEC proteins directly from whole lungs while leaving the basement membrane intact and as such differs from previous studies of BMP signalling in lungs that have been limited to analysis of whole lung lysates [6,7]. Western blotting of these lysates revealed that PCNA levels were significantly increased 1.8-fold in AECs in 1-NN-treated animals at $96 \mathrm{~h}$ compared to controls (Fig. 1C). Immunofluorescence for PCNA in lung tissue sections was also carried out. When present in the airway epithelium, PCNA was localised to the nuclei of AECs. Low numbers of PCNA-positive epithelial cells were evident in control airways at all time points (data not shown). An increased incidence of PCNA-positive cells was observed in 1-NN treatment groups at the 96 hour time point compared to control lungs, consistent with the lung lysis lavage data (Fig. 1D).

Thus we confirmed that in this acute injury model, inflammation preceded airway denudation (at $6 \mathrm{~h}$ and $24 \mathrm{~h}$ post 1-NN respectively). Importantly, we established here that airway re-epithelialisation was underway at $48 \mathrm{~h}$ and that proliferation did not occur until the later $96 \mathrm{~h}$ time point. This indicates that re-epithelialisation at $48 \mathrm{~h}$ following acute injury is primarily due to cell migration rather than proliferation.

\subsection{ERK and Smad activation in airway epithelial cells following 1-NN injury}

Having established the chronology of injury, inflammation, reepithelialisation and proliferation, we wanted to identify signalling pathways involved in airway regeneration following acute injury, and the time course of their activation. As a mediator of a range of signalling pathways including BMPs, the activation of ERK- $1 / 2$ was examined in lung lysates from control and 1-NN-treated animals. Involvement of the canonical BMP signalling pathway was also investigated.

At $6 \mathrm{~h}$ post exposure, levels of phosphorylated ERK-1 and ERK-2 (p-ERK-1 and p-ERK-2) were upregulated 3.5-fold and 10-fold respectively in AECs of 1-NN-treated animals compared with controls (Fig. 2A, B). At subsequent time points, levels of p-ERK-1 and p-ERK-2 were lower in 1-NN-treated AECs than in control cells.

Specific BMP signalling was also activated in lungs of 1-NN-treated rats. An antibody which detects phosphorylated Smads 1, 5 and/or 8 (p-Smad1/5/8) was used to assess Smad phosphorylation status. p-Smad $1 / 5 / 8$ was detected in AEC lysates from both control and 1-NNtreated animals at all time points (Fig. 2A). At 6 h post 1-NN treatment, p-Smad1/5/8 levels were significantly increased 0.6-fold in AECs of animals exposed to 1-NN compared to controls (Fig. 2A, B). At the later time points, $\mathrm{p}$-Smad1/5/8 levels were not significantly different between treated and control animals.

Localisation of $\mathrm{p}-\mathrm{Smad} 1 / 5 / 8$ was also examined by immunofluorescence in lung tissue sections. Consistent with the lung lysis lavage analysis, p-Smad1/5/8 was present in AECs of control animals and increased levels of p-Smad1/5/8 immunostaining were evident in AECs at $6 \mathrm{~h}$ in lungs from 1-NN-treated animals compared with controls (Fig. 2C).

\subsection{Airway epithelial cells are responsive to BMP4}

We next sought evidence as to which specific BMP ligand may be involved in activating Smad signalling in AECs. While expression of BMP4 has been reported in healthy and inflamed airway epithelial cells [7], it has not yet been confirmed whether normal AECs are in fact responsive to this BMP ligand. Rather than autocrine epithelial signalling, AECs may be non-responsive to the BMP4 they produce and mesenchymal cells may be the target for paracrine BMP4 signals emanating from the epithelium. Furthermore, the consequences of any such BMP4-mediated signalling in AECs are unknown.

We examined whether BMP4 was capable of activating Smad proteins in primary airway epithelial cells. Expression and nuclear localisation of Smad5, Smad8, p-Smad1/5/8 and Smad4 were examined by immunofluorescence. In the first instance, Smad expression was assessed in AECs in the absence of exogenous BMPs. All Smads were detected in these untreated cells (Fig. 3Aa-d). Smad5 was predominantly cytoplasmic with a low incidence of nuclear localisation. Smad8 was predominantly cytoplasmic in untreated AECs. p-Smad1/5/8 was predominantly localised to the nuclei of untreated AECs while Smad4 was cytoplasmic. The presence of $p-S \operatorname{mad} 1 / 5 / 8$ in the nucleus of untreated cells indicates that these cells express BMPs and respond to them in an autocrine manner. The expression of these Smad proteins was examined after $20 \mathrm{~min}$ and $2 \mathrm{~h}$ exposure to $100 \mathrm{ng} / \mathrm{ml} \mathrm{BMP4}$. Cytoplasmic levels of Smad5 were increased following 20 min exposure to BMP4 and increased nuclear localisation of both Smad5 and Smad8 was also apparent in BMP4-treated cells (Fig. 3Ae,f). The incidence of nuclear localised p-Smad1/5/8 did not appear upregulated after 20 min or $2 \mathrm{~h}$ exposure to BMP4 but was increased after $17 \mathrm{~h}$ (not shown). Smad4 was predominantly localised to the nucleus of AECs treated with BMP4 for $2 \mathrm{~h}$ (Fig. 3Ah).

Increased p-Smad1/5/8 expression levels in AECs in response to BMP treatment were also detected by Western blotting. Levels of p-Smad1/5/8 were upregulated 0.5-fold in AECs exposed to $100 \mathrm{ng} / \mathrm{ml}$ BMP4 for $72 \mathrm{~h}$ (Fig. 3B and C).

3.4. BMP4 alters morphology and downregulates E-cadherin expression in primary airway epithelial cells

We have reported previously that BMP4 induces a mesenchymallike morphology and downregulates E-cadherin expression in BEAS-2B transformed human airway epithelial cells [8]. However, the effects of BMP4 on normal AECs, including effects on E-cadherin expression, are unknown. We therefore examined the effect of BMP4 on morphology and E-cadherin expression in normal primary AECs.

Primary AECs cultured in control medium displayed characteristic epithelial 'cobblestone' morphology (Fig. 4Aa). In contrast, AECs cultured with $100 \mathrm{ng} / \mathrm{ml} \mathrm{BMP4}$ exhibited marked change in morphology with many cells appearing elongated and scattered with a mesenchymal-like morphology (Fig. 4Ab).

The effect of BMP4 on E-cadherin expression in these cells was examined. Immunofluorescence revealed that E-cadherin expression was downregulated at cell membranes of AECs cultured with BMP4 compared with untreated cells (Fig. 4Ac, d). Western blotting confirmed that E-cadherin levels were decreased in BMP4-treated cells to $50 \%$ of the level observed in untreated cells (Fig. 4B and C). The E-cadherin antibody used in this study recognises both the full length E-cadherin $(120 \mathrm{kDa})$ and a truncated form of the protein $(\sim 33 \mathrm{kDa})$. This truncated form represents posttranslational regulation of Ecadherin-mediated adherens junction stability [18] and is often observed in E-cadherin Western blots. Levels of this truncated form of E-cadherin were reduced in proportion to those of full length Ecadherin following BMP4 treatment (Fig. $4 \mathrm{~B}$ and C). This suggests that the reduction in levels of full length E-cadherin in response to BMP4 was not due to an increase in posttranslation cleavage of the protein, a possible mechanism for downregulation of E-cadherin [18].

Quantitative PCR was therefore carried out to determine whether BMP4 induced transcriptional downregulation of E-cadherin. Ecadherin mRNA expression was reduced 0.28 -fold and 1.58 -fold in AECs in response to 50 and $100 \mathrm{ng} / \mathrm{ml} \mathrm{BMP4}$ respectively (Fig. 4D).

We and others have shown that BMP4 upregulates transcriptional repressors of E-cadherin including Snai1 $[8,19]$ but this has not been demonstrated to date in normal AECs. We therefore examined the effects of BMP4 on Snai1 expression in these cells. Snai1 expression was elevated in AECs in response to $100 \mathrm{ng} / \mathrm{ml} \mathrm{BMP4}$. 
A
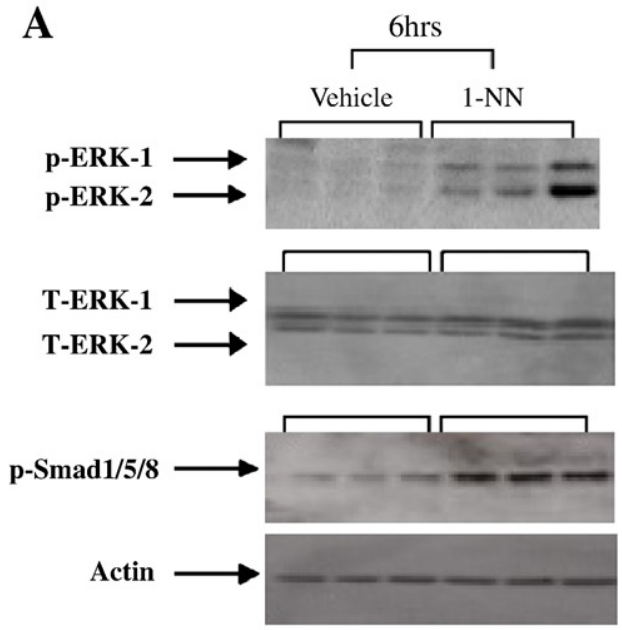

B

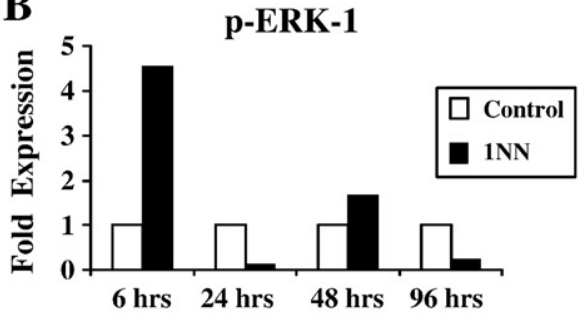

C

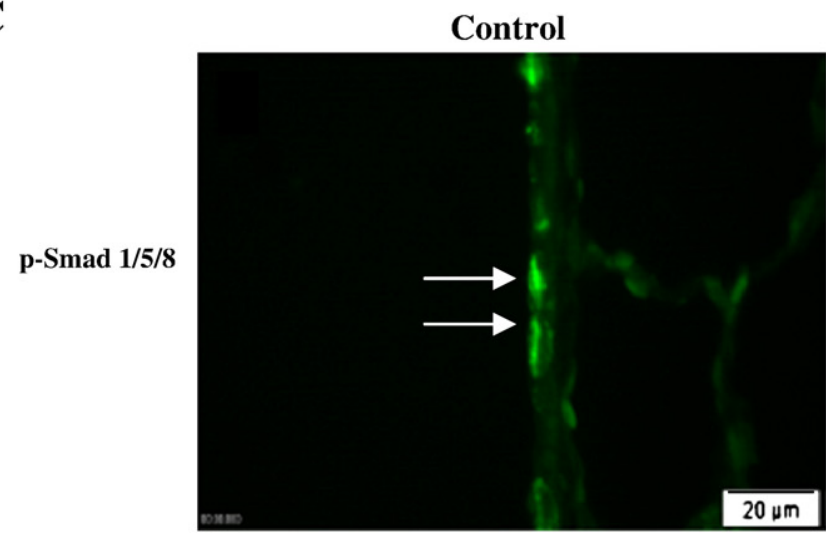

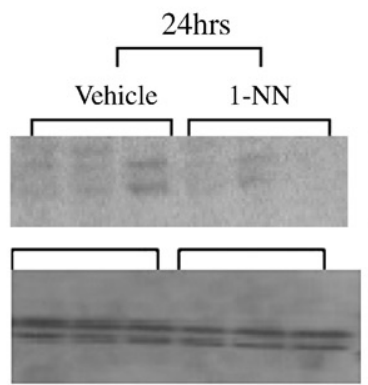
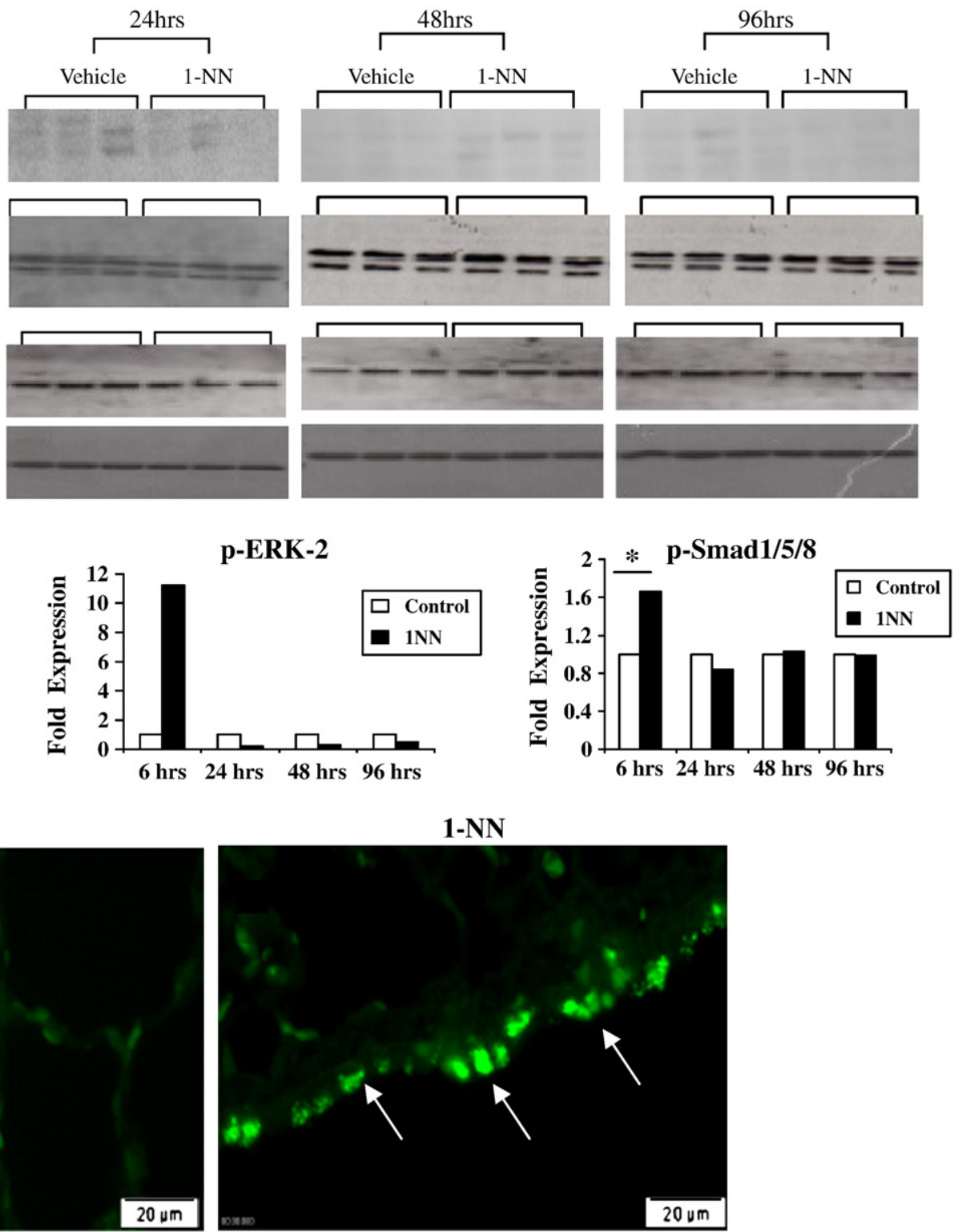

Fig. 2. Expression of signalling proteins in airway epithelium of control and 1-NN-treated animals. (A) Western blots of lung lysis lavage from control vehicle-treated ( $\mathrm{n}=3$ ) and 1-NNtreated $(n=3)$ animals showing expression of signalling molecules in airway epithelial cells. (B) Densitometric analysis of blots showed that levels of p-ERK-1 and p-ERK-2 were increased at $6 \mathrm{~h}$ post 1-NN treatment compared to vehicle controls. Levels of p-Smad1/5/8 were also increased at $6 \mathrm{~h}$ in 1 -NN injured airway epithelium compared with vehicle controls. p-ERK-1 and p-ERK-2 were normalised to total ERK-1 and ERK-2 (T-ERK-1 and T-ERK-2) respectively and p-Smad1/5/8 was normalised to actin. (C) Immunofluorescence revealed p-Smad1/5/ 8 expression in control airway epithelial cells and increased expression in cells from 1-NN-treated animals at 6 h. All densitometry data represent $\mathrm{n}=3$ animals. Significance compared to controls was obtained using the Student's $t$ test $\left({ }^{*} \mathrm{P}<0.05\right)$. Scale bars represent $20 \mu \mathrm{m}$.

3.5. BMP4 enhances migration of airway epithelial cells in a wound closure assay

Given that in vivo in 1-NN injured airways, increased Smad signalling coincides with the migration phase of regenerating airways and that in vitro, BMP4 induced a mesenchymal-like morphology and downregulated E-cadherin in primary AECs, we used a novel wound closure assay to determine whether BMP4 enhances migration of these cells. In this assay, AECs are seeded into multiwell plates onto an outer ring area coated with collagen type IV and allowed to become confluent. The centre of the ring has been coated with fibronectin to mimic a wound area and is covered with a stopper to prevent cell attachment. When the cells in the outer ring are confluent, the stopper is removed to expose the fibronectin 'wound' and the cells are allowed to migrate.
BMP4 increased the rate of migration of cells into the wound area. The number of cells in the wound area was 0.77 -fold greater $(n=3)$ in the presence of BMP4 than that in control SFM $24 \mathrm{~h}$ after removal of the stopper (Fig. 5A and B). Migration in the presence of BMP4 was also slightly enhanced compared to the $10 \%$ FCS positive control.

\section{Discussion}

To date, understanding of the expression of BMP signalling pathway components in normal, injured and diseased lungs has been very limited and the role of BMP signalling in normal airway epithelial cells has been unknown. We have previously shown that BMP4 downregulates Ecadherin expression and increases motility of transformed airway epithelial cells [8]. We subsequently hypothesised that, if replicated in vivo and in primary airway cells, these effects of BMP signalling could 
A

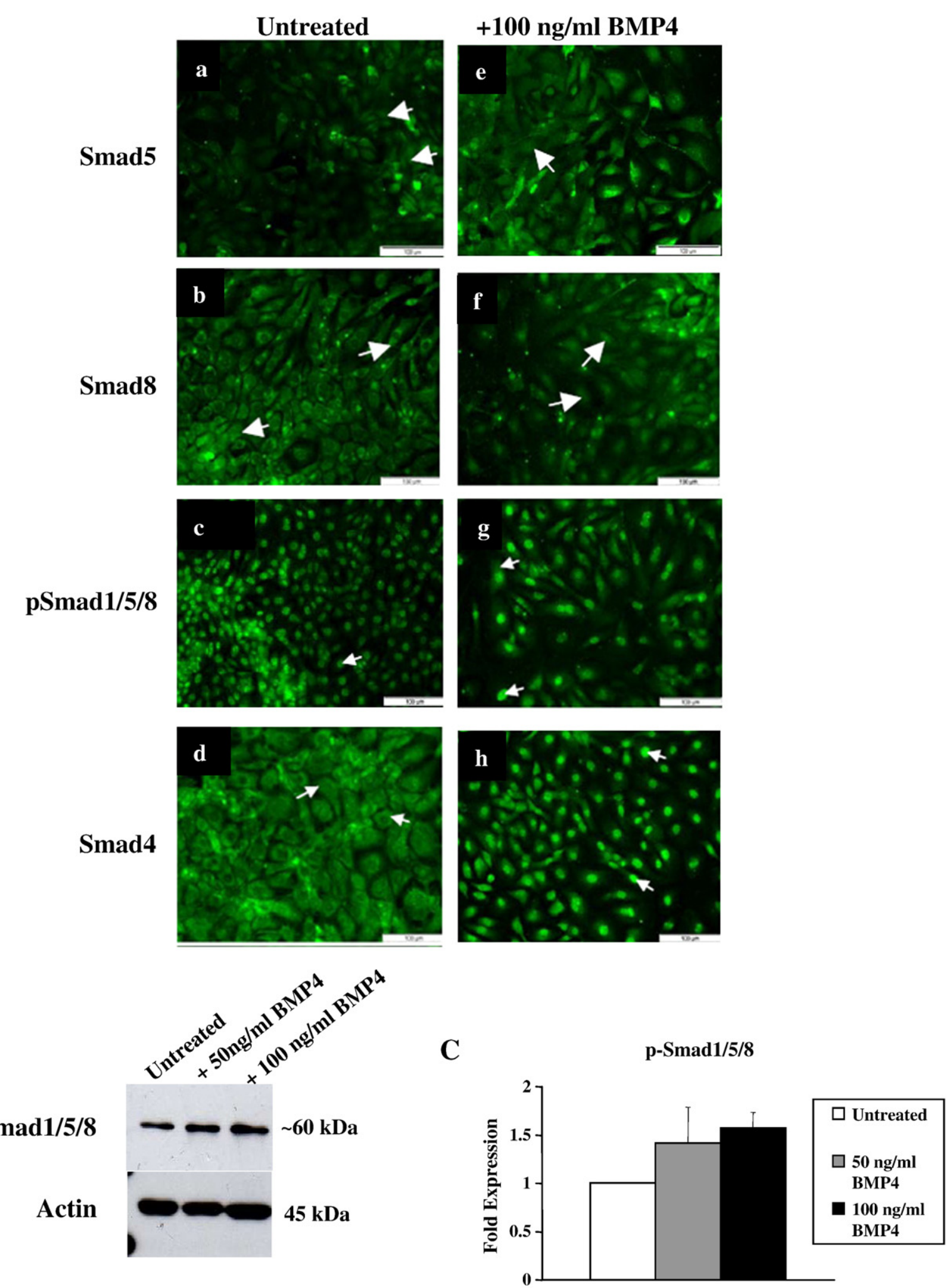

Fig. 3. BMP4 activates Smad signalling in normal airway epithelial cells. (A) Representative photomicrographs of Smad protein expression in untreated cells and cells treated with $100 \mathrm{ng} / \mathrm{ml} \mathrm{BMP4}$ for $20 \mathrm{~min}$ or $2 \mathrm{~h}$. Nuclear (arrows) and/or cytoplasmic expression of Smad5, Smad8, p-Smad1/5/8 and Smad4 was detected in normal AECs in the absence of exogenous BMP ligand (a-d). Increased incidence of nuclear localisation of all Smads was observed following addition of BMP4 (e-h). Scale bar represents 100 um. (B) Western blotting and $(C)$ densitometric analysis ( $n=2$, normalised to actin) confirmed that levels of $\mathrm{p}$-Smad1/5/8 remained elevated in normal AECs cultured in the presence of $50 \mathrm{ng} / \mathrm{ml}$ or $100 \mathrm{ng} / \mathrm{ml} \mathrm{BMP} 4$ for $72 \mathrm{~h}$.

play an important role in normal and disease processes including regeneration, inflammation and carcinogenesis. In the present study, we examined epithelial regeneration, utilising the rat $1-\mathrm{NN}$ acute injury model which has been used previously primarily for toxicity studies and in which the timeframe of airway epithelial regeneration was unknown. Our study provides novel insight into the chronology of regeneration 
A
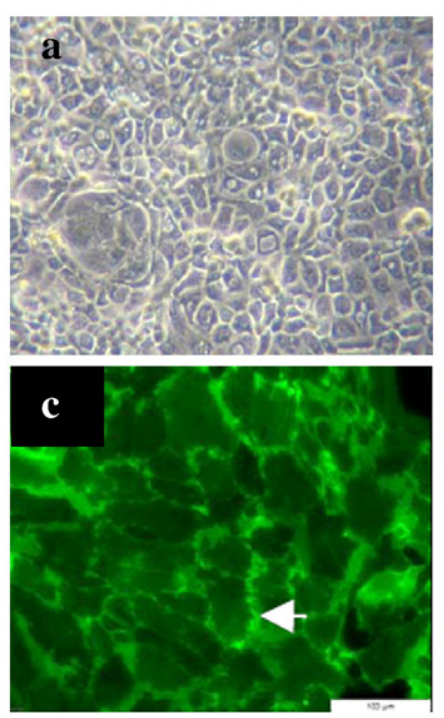

+100 ng/ml BMP4
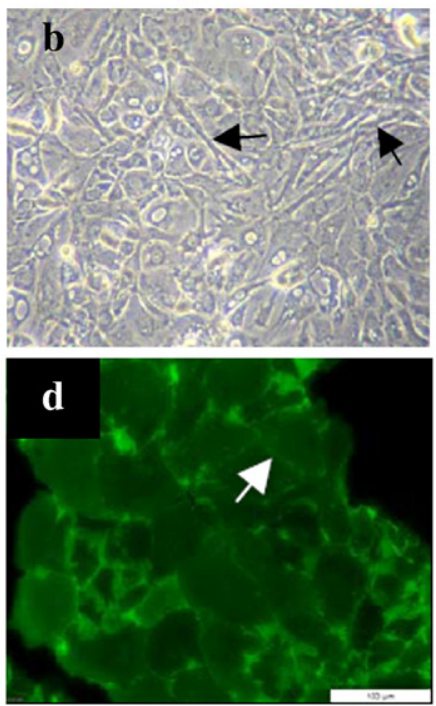

C

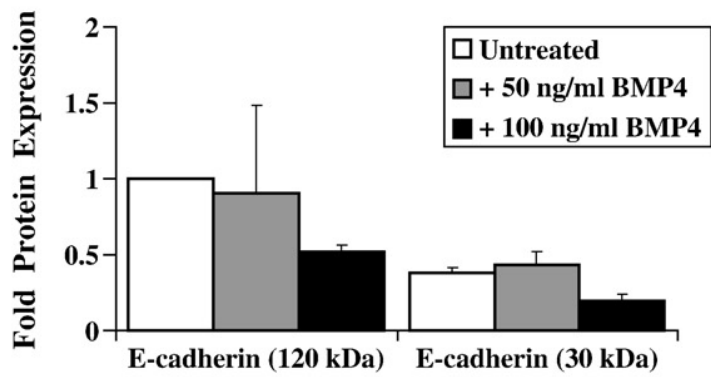

D

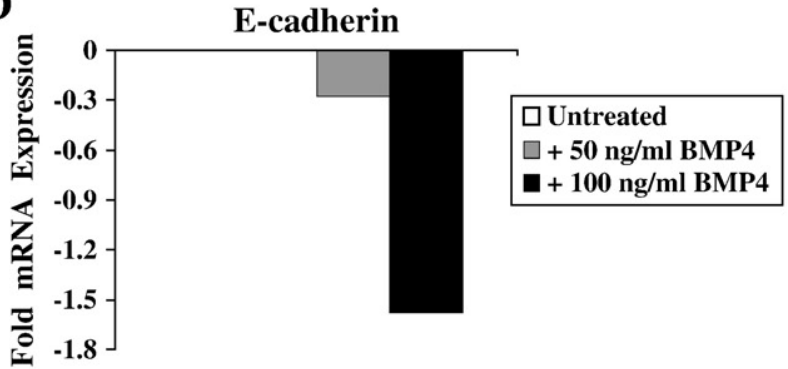

B

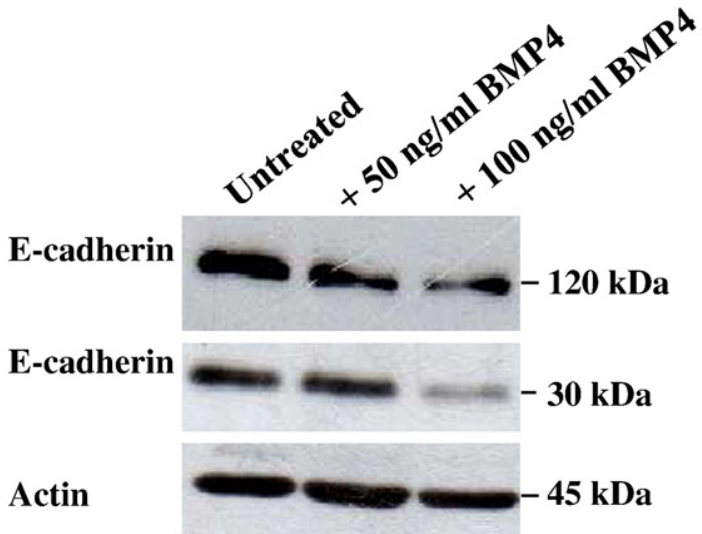

$\mathbf{E}$

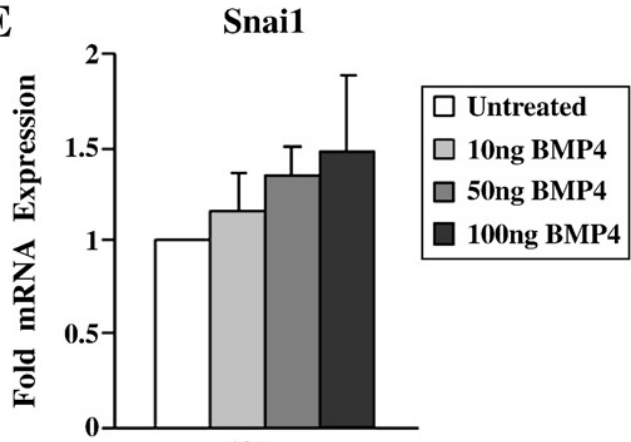

Fig. 4. Effect of BMP4 on morphology and E-cadherin expression in normal airway epithelial cells. (A) Untreated AECs showed a typical epithelial morphology (a) while AECs cultured with $100 \mathrm{ng} / \mathrm{ml} \mathrm{BMP} 4$ for $72 \mathrm{~h}$ displayed an altered morphology with cells acquiring an elongated mesenchymal-like appearance (b) and immunofluorescence revealed that E-cadherin expression was reduced at regions of cell-cell contact in these cells (c, d). (B) and (C) Western blotting and densitometry ( $\mathrm{n}=2$ ) confirmed that E-cadherin protein levels were reduced in AECs cultured with BMP4 for $72 \mathrm{~h}$. A concomitant reduction in a $30 \mathrm{kDa}$ truncated form of E-cadherin also occurred. (D) Quantitative PCR revealed that mRNA levels of E-cadherin were reduced in AECs cultured with BMP4 for $48 \mathrm{~h}(\mathrm{n}=2)$. (E) Semi-quantitative RT-PCR showed that Snai1 expression was increased in AECs cultured with BMP4 for $48 \mathrm{~h}(\mathrm{n}=2$ except $100 \mathrm{ng} / \mathrm{ml}$ BMP4 $\mathrm{n}=4)$.

events that follow acute airway injury and the involvement of BMP signalling in regeneration in vitro and in vivo.

Our observations of maximal inflammation and airway damage at $6 \mathrm{~h}$ and $24 \mathrm{~h}$ respectively are consistent with previous studies concerned with the toxicology of 1-NN. The airway cell types affected by 1 -NN-mediated toxicity at $24 \mathrm{~h}$ post exposure have been described previously in a detailed histopathological evaluation of 1-NN toxicity in rat lungs. At doses of $50 \mathrm{mg} / \mathrm{kg} 1-\mathrm{NN}$, ciliated cell toxicity is minimal while nonciliated (Clara) cell loss is equally prevalent in all airway levels resulting in a discontinuous epithelium with focal denudation of the basement membrane [13]. In our study, we now demonstrate that $1-\mathrm{NN}$-induced inflammation coincides with early elevation of ERK and Smad1/5/8 signalling activity in AECs, and significantly, that this occurs prior to airway denudation. We also show that re-epithelialisation occurs by $48 \mathrm{~h}$ post 1 -NN administration. The fact that cell proliferation was maximal at $96 \mathrm{~h}$ post $1-\mathrm{NN}$ exposure indicates that re-epithelialisation is mainly due to migration of remaining epithelial cells rather than proliferation. This is consistent with current understanding that a cell migration phase precedes a proliferation phase during airway epithelial regeneration [20].

The two previous studies of BMP signalling pathway components in mouse and human inflamed airways used Western blotting of whole lung lysate and immunostaining to examine protein expression and reported differing observations of p-Smad1/5 in normal airway epithelial cells [6,7]. In contrast, our use of the lung lysis lavage technique combined with immunofluorescence for this study has allowed us to precisely localise and quantify signalling molecules in AECs in vivo. With both techniques, we observed p-Smad1/5/ 8 immunoreactivity in both normal and regenerating rat airway epithelium, consistent with the previous study of human asthmatic airways [7]. Thus, unlike ERK signalling, the canonical BMP Smad signalling pathway is constitutively active in healthy airway epithelial cells, indicating an as yet unknown role in maintenance of cell 
A

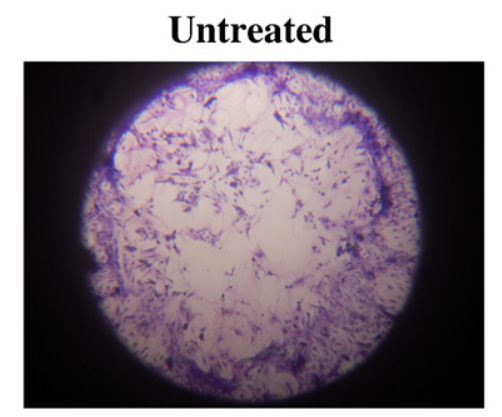

+100 ng BMP4

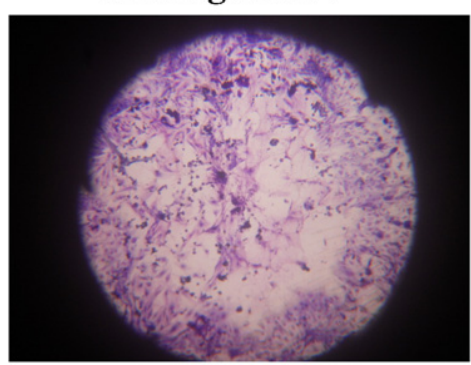

$+10 \%$ FCS

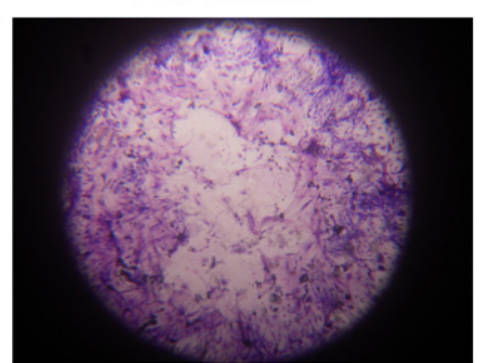

B

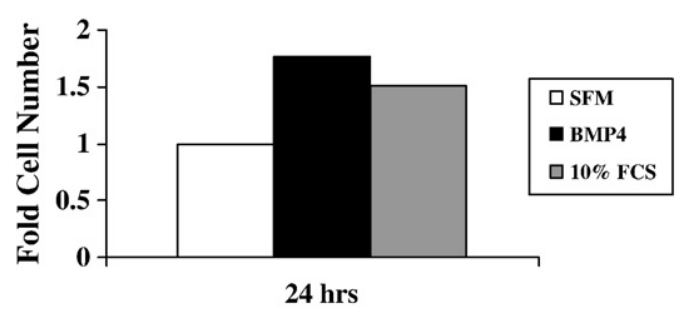

Fig. 5. BMP4 induces migration of normal airway epithelial cells. (A) Representative photomicrographs of wound closure assay showing airway epithelial cell migration onto fibronectin-coated 'wound' area after $24 \mathrm{~h}$. Black region is detection mask used to delineate wound area. (B) The number of cells in the wound area was 0.77 -fold greater ( $\mathrm{n}=3$ ) in the presence of BMP4 than that in control SFM after 24 h. Photomicrographs at $20 \times$ magnification.

function. It is not yet clear which cell types within the epithelium possess this constitutive Smad activity and if the increase following injury occurs in these same cells or in another subpopulation.

The elevation of ERK and Smad1/5/8 activation at the 6 hour time point, before epithelial injury was evident histologically at $24 \mathrm{~h}$, suggests that their respective pathways play key early roles in the regeneration process. This signalling coincided with the period of inflammatory cell infiltration. Therefore, inflammation, rather than tissue damage, may be the critical signal for activation of a regeneration response in injured AECs. However, there may be a two-way communication between inflammatory cells and epithelial cells. We reported previously that vascular cell adhesion molecule 1 (VCAM1) mRNA is highly upregulated in transformed airway epithelial cells stimulated with BMP4 [8]. VCAM1 is involved in leukocyte migration during inflammation [21]. We have not examined VCAM1 expression in the present study but if BMP4 has a similar effect on airway cells in vivo, this could be a mechanism whereby BMP signalling activates airway epithelial cells to increase inflammatory cell infiltration following injury.

BMP4 is expressed in epithelial cells in the tips of growing mouse lung buds [5] and is reportedly expressed throughout the airway epithelium, in submucosal cells and in inflammatory cells in adult human airways [7]. In the mouse and human allergic lung inflammation studies, levels of BMP4 did not increase during inflammation $[6,7]$. This suggests that constitutively expressed BMP4, probably from damaged epithelial cells and possibly from local inflammatory cells, is a likely source of BMP ligand in our 1-NN acute injury model. Importantly, the demonstration that nuclear $\mathrm{p}-\mathrm{Smad} 1 / 5 / 8$ is present in untreated normal AECs here (Fig. 3Ac) indicates that these cells produce BMPs and are responsive in an autocrine manner. BMP4 is critical to a variety of developmental processes, including EMT aspects of morphogenesis, and transcriptional repression of E-cadherin is a key step in EMT [22]. The use of primary AEC cultures enabled us to demonstrate for the first time that normal AECs are responsive to BMP4 and that a consequence of BMP4 signalling in these cells is the downregulation of E-cadherin mRNA and protein expression. Therefore, we have shown that the ability of BMP4 to downregulate epithelial-related proteins during morphogenesis is recapitulated in normal adult airway epithelial cells. We therefore suggest that the role of BMP4 as lung morphogen during development is recapitulated, at least to some degree, in adult lungs when airways undergo regeneration following injury. The combined data from this study and our previous study [8] suggest that in injured airways in vivo, BMP4 signalling in AECs is an early event following injury, resulting in migration of epithelial cells across the denuded basement membrane and that downregulation of E-cadherin may be required for this process. It is conceivable that in vivo, inappropriately sustained BMP signalling, possibly as a result of chronic injury/inflammation, could result in a prolonged decrease in E-cadherin expression in the airways. This in turn could compromise epithelial barrier integrity in the short term and could possibly lead to remodelling, EMT or tumourigenesis in the longer term.

In summary, we have demonstrated that BMP signalling is active in the adult airway epithelium during regeneration and that BMP4 alters the morphology, downregulates E-cadherin expression and induces migration in normal airway epithelial cells. Our data suggest that the role played by BMPs in controlling tissue architecture during development is recapitulated in adult airways during regeneration after injury.

\section{Author contributions}

JCM: designed and carried out animal study, harvested tissues, carried out BAL analysis and contributed to manuscript preparation.

ELM: designed and carried out primary airway epithelial cell experiments and contributed to manuscript preparation.

JLG: carried out Western blotting, densitometry and immunofluorescence for animal work and contributed to manuscript preparation.

NMcC: designed and carried out wound closure assay and analysis.

AA: designed and carried out QPCR assay.

SOD: won grant funding, provided hypothesis, directed all work, wrote manuscript and prepared figures.

\section{Acknowledgements}

This work was funded by European Union Marie Curie research grant MTKD-CT-2004-014436 and Science Foundation Ireland research grant 
02/IN.1/B52. The authors thank Dr. Alan Buckpitt, UC Davis School of Veterinary Medicine, USA, for his assistance in carrying out the 1-NN studies and for his comments during manuscript preparation.

\section{References}

1] W. Shi, J. Xu, D. Warburton, Respirology 14 (2009) 656

2] D. Chen, M. Zhao, S.E. Harris, Z. Mi, Front. Biosci. 9 (2004) 349.

[3] G. Winnier, M. Blessing, P.A. Labosky, B.L. Hogan, Genes Dev. 9 (1995) 2105.

[4] D. Obradovic Wagner, C. Sieber, R. Bhushan, J.H. Borgermann, D. Graf, P. Knaus, Sci. Signal. 3 (2010) 1.

[5] M. Weaver, J.M. Yingling, N.R. Dunn, S. Bellusci, B.L. Hogan, Development 126 (1999) 4005

[6] A. Rosendahl, E. Pardali, M. Speletas, P. Ten Dijke, C.H. Heldin, P. Sideras, Am. J. Respir Cell Mol. Biol. 27 (2002) 160

[7] H.H. Kariyawasam, G. Xanthou, J. Barkans, M. Aizen, A.B. Kay, D.S. Robinson, Am. J Respir. Crit Care Med. 177 (2008) 1074.

[8] E.L. Molloy, A. Adams, J.B. Moore, J.C. Masterson, L. Madrigal-Estebas, B.P. Mahon, S. O'Dea, Growth Factors 26 (2008) 12.

[9] N.G. Kan, D. Junghans, J.C. Izpisua Belmonte, FASEB J. 23 (2009) 3516.
[10] I.U. Mysorekar, M. Isaacson-Schmid, J.N. Walker, J.C. Mills, S.J. Hultgren, Cell Host Microbe 5 (2009) 463.

[11] A.M. Wheelock, L. Zhang, M.U. Tran, D. Morin, S. Penn, A.R. Buckpitt, C.G. Plopper Am. J. Physiol. 286 (2004) L399.

[12] J.L. Gilbert, J. Purcell, P. Strappe, M. McCabe, T. O'Brien, S. O'Dea, Anticancer Drugs 19 (2008) 783.

[13] R. Paige, V. Wong, C.G. Plopper, Toxicol. Appl. Pharmacol. 147 (1997) 224.

[14] L.S. Van Winkle, A.R. Buckpitt, S.J. Nishio, J.M. Isaac, C.G. Plopper, Am. J. Physiol. 269 (1995) L800.

[15] A.C. Zemke, J.C. Snyder, B.L. Brockway, J.A. Drake, S.D. Reynolds, N. Kaminski, B.R. Stripp, Am. J. Respir. Cell Mol. Biol. 40 (2009) 340.

[16] C.Y. Lin, A.M. Wheelock, D. Morin, R.M. Baldwin, M.G. Lee, A. Taff, C. Plopper, A. Buckpitt, A. Rohde, Toxicology 260 (2009) 16.

[17] J. Sauer, R. Eversole, C. Lehmann, D. Johnson, L. Beuving, Toxicol. Lett. 90 (1997) 19.

[18] J. Masterson, S. O'Dea, Cells Tissues Organs 185 (2007) 175.

[19] B.L. Theriault, T.G. Shepherd, M.L. Mujoomdar, M.W. Nachtigal, Carcinogenesis 28 (2007) 1153.

20] C.G.A. Persson, J.S. Erjefalt, I. Erjefalt, M.C. Korsgren, M.C. Nilsson, F. Sundler, Clin. Exp. Allergy 26 (1996) 746.

[21] E.S. Wittchen, Front. Biosci. 14 (2009) 2522

[22] L. Larue, A. Bellacosa, Oncogene 24 (2005) 7443. 